\title{
A Study Evaluating the effects of Throat Packs during Nasal Surgery- A Randomised Controlled Trial
}

\section{Ali Al-lami, Emma McClelland, Kwamena Amonoo-Kuofi, Praneta Kulloo, Raj Lakhani, Navin Prakash, Nazir Bhat}

\section{Introduction}

Water-soaked gauze throat packs are utilised in a large number of rhinological procedures in Ear, Nose \& Throat (ENT) departments worldwide.

The role of a throat pack is primarily to prevent contamination of the aerodigestive tract with blood and secretions. This theoretically prevents airway morbidity and also minimises post-operative nausea and vomiting (PONV) as a result of blood entering the stomach via the oesophagus.

It is well recognised that the use of throat packs can result in significant post-operative throat pain. Various studies suggest an incidence ranging from $15 \%-61 \%$ [1-3]. There appears to be no correlation with duration of packing or number of intubations [4]. No trials have satisfactorily investigated the correlation between using a throat pack and post-operative nausea and vomiting [5]

A recent literature review highlighted the deficiencies in previous studies examining this topic. The review highlighted the paucity of adequately powered, well designed trials investigating the relationship between the use of throat packs and post-operative morbidity in terms of pain and PONV.

The study aims were:

Primary outcome measure was difference in postoperative nausea and vomiting (PONV) between patients with and without throat packs during nasal surgery

Our secondary outcome measure was difference in throat pain levels in the same two groups.

\section{Methods}

Double Blind, prospective, randomised controlled trial.

Included: Age $>18$, FESS, septoplasty, septorhinoplasty, RIT, Nasal polypectomy.

Excluded: nasal surgery for malignant disease, and patients with history of post-operative nausea and vomiting.

1.Subjects are identified from ENT clinic and added to the waiting list to undergo surgery. 2.Subjects are invited to participate in study, counselled and given an information sheet 3.At Induction patients are randomised to receive either throat pack or not via presealed envelopes containing prearranged random sequence (surgeon blinded)

4.Patients and assessors complete questionnaire detailing degree of pain/ discomfort performed in recovery when awake, 2 and 6 hours post op (assessors blinded)

5.Patients and assessors complete questionnaire detailing degree of nausea and vomiting at 6 hours post op. 6.Patients followed up as per normal post op protocol.

7.Results of clinical findings collated, anonymised and analysed

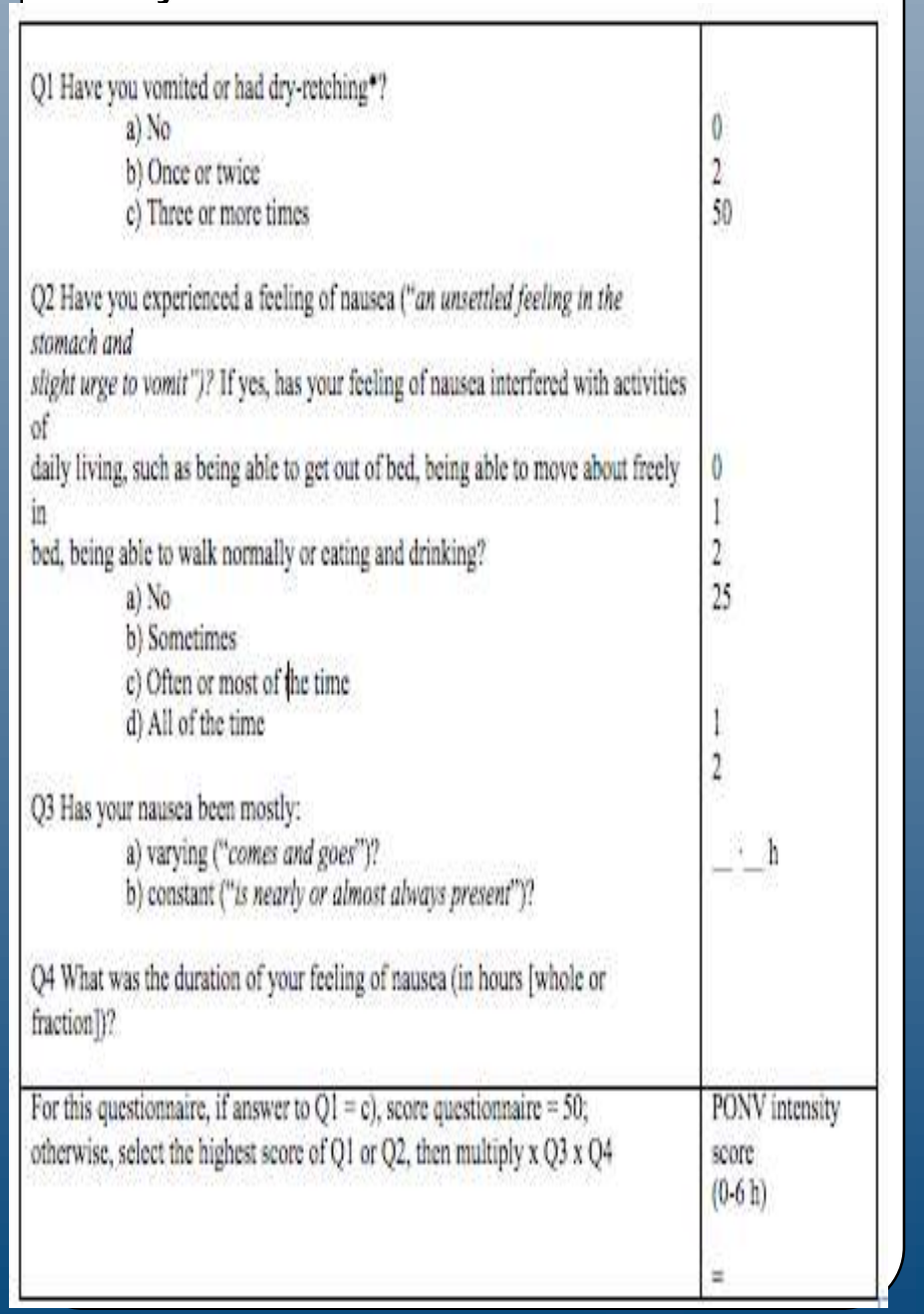

\section{Results}

Demographics and operations categories

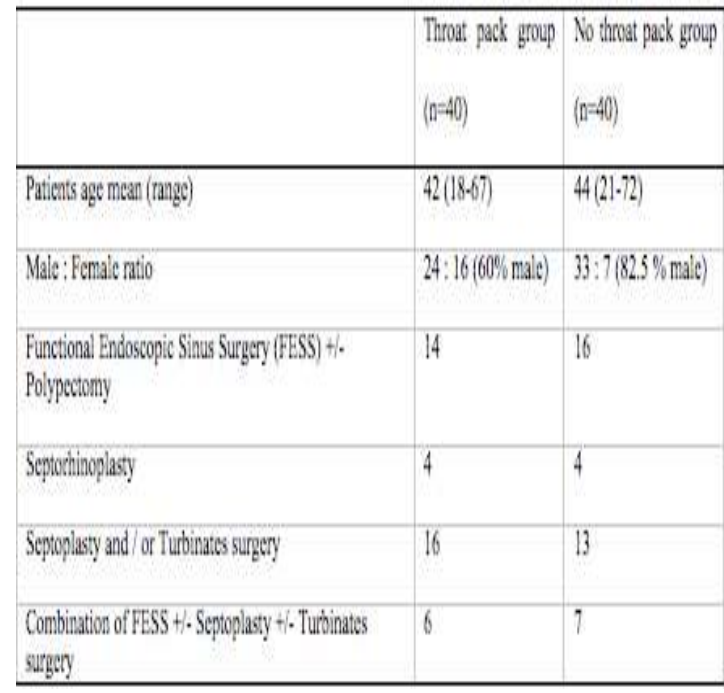

Statistical analysis
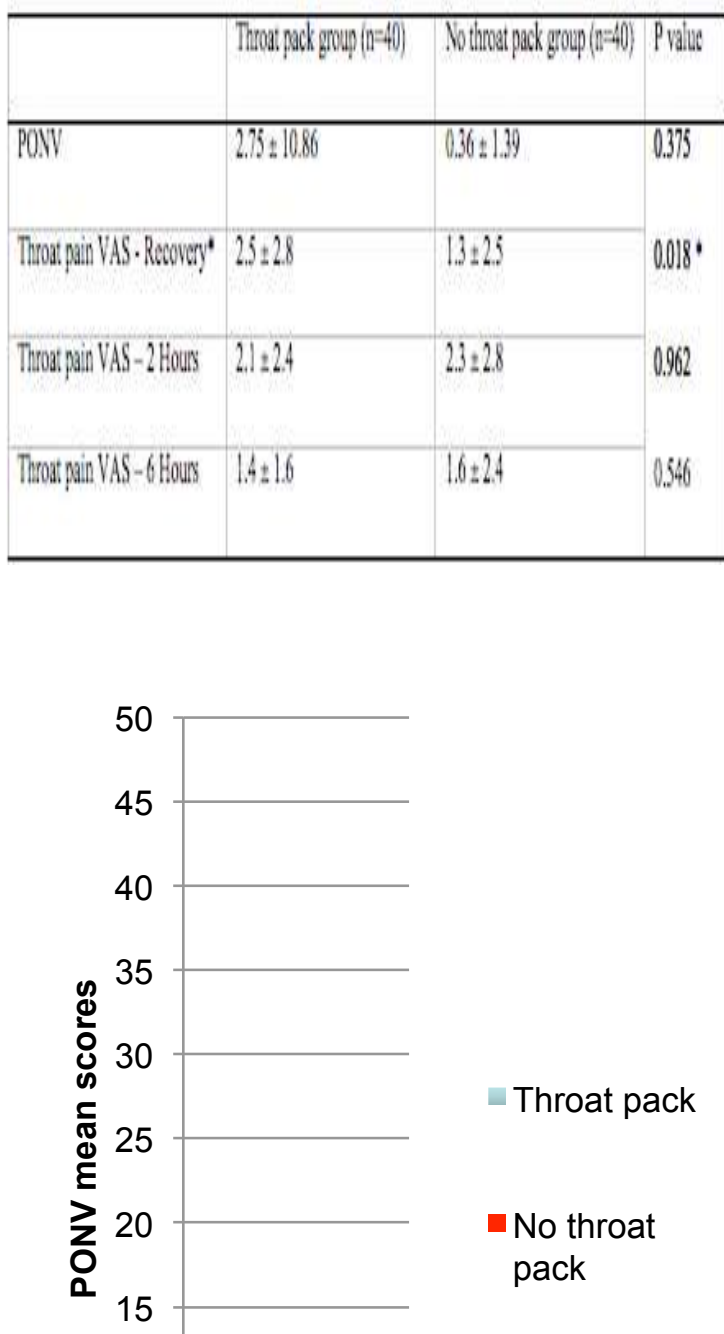

10

5

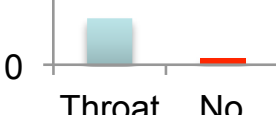
Throat No pack

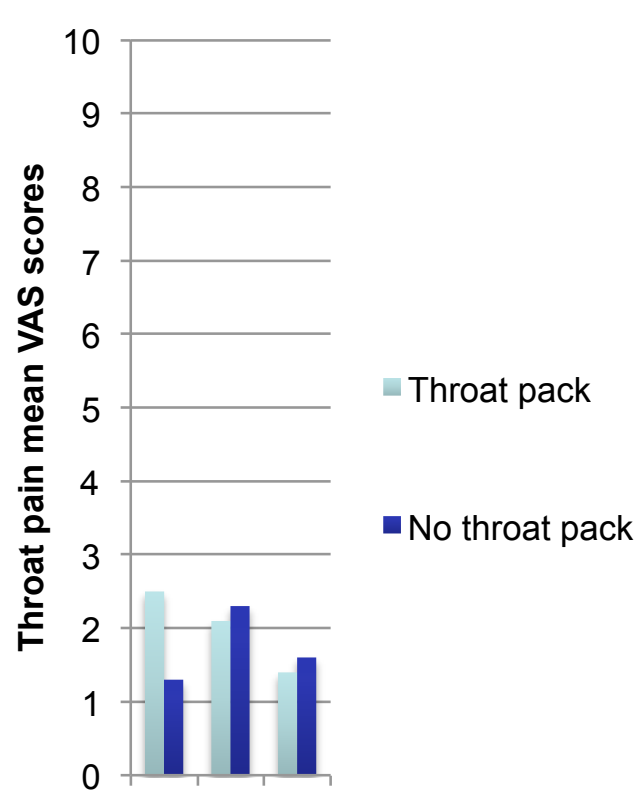

Conclusions

The important theoretical reasons of using the throat packs in nasal surgery include reduction in aspirated and ingested blood into the airway and oesophagus respectively.

There were no airway morbidity events noted during this study.

The lack of statistically significant difference in PONV intensity score contradicts the theoretical reason for using throat packs in nasal surgery.

With regards to throat pain, although small in terms of difference, the only statistically significant event in this study was that throat pain in recovery was higher in the throat pack group.

In light of the results of this study, weighing up the theoretical reasons for using throat packs whilst considering minimising the patient discomfort in the recovery period are important considerations in the decision whether to use throat packs or not.

Furthermore, with the current drive in performing most rhinological procedures as day case surgeries for patient benefits, financial and case turnover reasons, the use of throat packs could potentially delay the patient discharge due to throat pain

\section{Bibliography}

1] Conway CM, Miller JS, Sugden FLH (1960) Sore throat after anaesthesia. Br J Anaesth 32:219 223.

[2] Edmuds-Seal J, Eve JH (1962) Minor sequelae anaesthesia: a pilot study. Br J Anaesth 34:44-48.

[3] Hartsell CJ, Stephen CR (1964) Incidence of sore throat following endotracheal intubation. Can J Anaesth 11:307-312.

[4] Jaiswal V, Bedford GC (2009) Review of nasal packs in nasal surgery. JLO 123:701-704.

[5] Colbert S, Jackson M, Turner M, Brennan PA (2012) Reducing the risk of retained throat packs after surgery. Br J Oral Maxillofac Surg 50:680-681. 